\title{
Seamless Translation of Optical Fiber PolMux-OFDM into a 2x2 MIMO Wireless Transmission Enabled by Digital Training-Based Fiber-Wireless Channel Estimation
}

Pang, Xiaodan; Zhao, Ying; Deng, Lei; Binti Othman, Maisara; Yu, Xianbin; Jensen, Jesper Bevensee; Zibar, Darko; Tafur Monroy, Idelfonso

\section{Published in:}

Proceedings of SPIE, the International Society for Optical Engineering

Link to article, DOI:

$10.1117 / 12.905495$

Publication date:

2011

Link back to DTU Orbit

Citation (APA):

Pang, X., Zhao, Y., Deng, L., Binti Othman, M., Yu, X., Jensen, J. B., Zibar, D., \& Tafur Monroy, I. (2011). Seamless Translation of Optical Fiber PolMux-OFDM into a 2x2 MIMO Wireless Transmission Enabled by Digital Training-Based Fiber-Wireless Channel Estimation. In Proceedings of SPIE, the International Society for Optical Engineering (Vol. 8309, pp. 83090C). SPIE - International Society for Optical Engineering. Proceedings of SPIE - The International Society for Optical Engineering https://doi.org/10.1117/12.905495

\section{General rights}

Copyright and moral rights for the publications made accessible in the public portal are retained by the authors and/or other copyright owners and it is a condition of accessing publications that users recognise and abide by the legal requirements associated with these rights.

- Users may download and print one copy of any publication from the public portal for the purpose of private study or research.

- You may not further distribute the material or use it for any profit-making activity or commercial gain

- You may freely distribute the URL identifying the publication in the public portal 


\title{
Seamless Translation of Optical Fiber PolMux-OFDM into a $2 \times 2$ MIMO Wireless Transmission Enabled by Digital Training-Based Fiber-Wireless Channel Estimation
}

\author{
Xiaodan Pang*a, Ying Zhao ${ }^{b}$, Lei Deng ${ }^{c}$, M. B. Othman ${ }^{a}$, Xianbin Yu $^{a}$, J. B. Jensen ${ }^{a}$, \\ D. $\operatorname{Zibar}^{a}$ and I.T. Monroy ${ }^{a}$ \\ ${ }^{a}$ DTU Fotonik, Technical University of Denmark, DK-2800, Kgs. Lyngby, Denmark \\ ${ }^{b}$ Department of Electronic Engineering, Tsinghua University, 10084, Beijing, China \\ ${ }^{c}$ School of Optoelectronics Science and Engineering, HuaZhong University of Science and Technology, Wuhan, \\ China
}

\begin{abstract}
We propose and demonstrate a $2 \times 2$ multiple-input multiple-output (MIMO) wireless over fiber transmission system. Seamless translation of two orthogonal frequency division multiplexing (OFDM) signals on dual optical polarization states into wireless MIMO transmission at $795.5 \mathrm{Mbit} / \mathrm{s}$ net data rate is enabled by using digital training-based channel estimation. A net spectral efficiency of $2.55 \mathrm{bit} / \mathrm{s} / \mathrm{Hz}$ is achieved.
\end{abstract}

Keywords: radio frequency photonics, wireless communication, polarization multiplexing, MIMO, OFDM

\section{INTRODUCTION}

Hybrid optical fiber-wireless transmission systems will play an important part in the next generation usercentered access networking. This truly user-centered network will be powered by free access to services and applications enabled by seamless broadband fiber-wireless connections to various devices nearby. To realize the seamless integration of wireless and fiber-optic networks, the wireless links needs to be developed to preserve transparency to bit-rates and modulation formats. ${ }^{1}$

Orthogonal frequency division multiplexing (OFDM) is a promising candidate signal format for future hybrid optical fiber-wireless access systems. This is because of the inherent high chromatic dispersion tolerance of OFDM signals in optical fibers and its robustness against frequency selective fading or narrowband interference in wireless channels. ${ }^{2,3}$ More interestingly, the spectral efficiency in fiber links can be further increased by polarization multiplexing (PolMux). ${ }^{4}$ Research on baseband PolMux OFDM transmission has been reported..$^{5,6}$ In wireless channels, multiple-input multiple-output (MIMO) technique provides an attractive solution to increase capacity for bandwidth limited systems. ${ }^{7}$ Meanwhile, the well-known radio-over-fiber (RoF) technology, which combines optical fiber and wireless techniques, provides a good solution to increase the coverage while maintaining the mobility of the broadband services in the local area networking scenarios. Based on these reasons, a RoF system combining optical PolMux, wireless MIMO and OFDM data format is able to fulfill the requirements of robustness, high flexibility and high spectral efficiency for providing broadband services in local networks. So far, in reported work focusing on PolMux wireless MIMO systems, only simple modulation format like on-off keying $(\mathrm{OOK})$ is used. ${ }^{7}$

In addition, for multi-carrier systems like OFDM, a large computational complexity will be introduced by using the classical MIMO channel estimation method based on the butterfly structure because an adaptive filter needs to be assigned for each OFDM subcarrier. Consequently, a training-based channel estimation method has the relatively low computational complexity at the receiver ${ }^{5}$ and draws more interests in analyzing multi-carrier systems.

\footnotetext{
* Send correspondence to Xiaodan Pang: E-mail: xipa@fotonik.dtu.dk, Telephone: +45 45257375
} 
In this paper, we experimentally demonstrate a proof-of-concept hybrid fiber wireless system. A PolMux 4-quadrature amplitude modulation (4-QAM) OFDM RoF signal is seamlessly translated into a $2 \times 2$ MIMO wireless transmission. By using training sequence-based OFDM-MIMO channel estimation, transmitted wireless signal in both branches are successfully demodulated and a net spectral efficiency of $2.55 \mathrm{bit} / \mathrm{s} / \mathrm{Hz}$ is achieved.

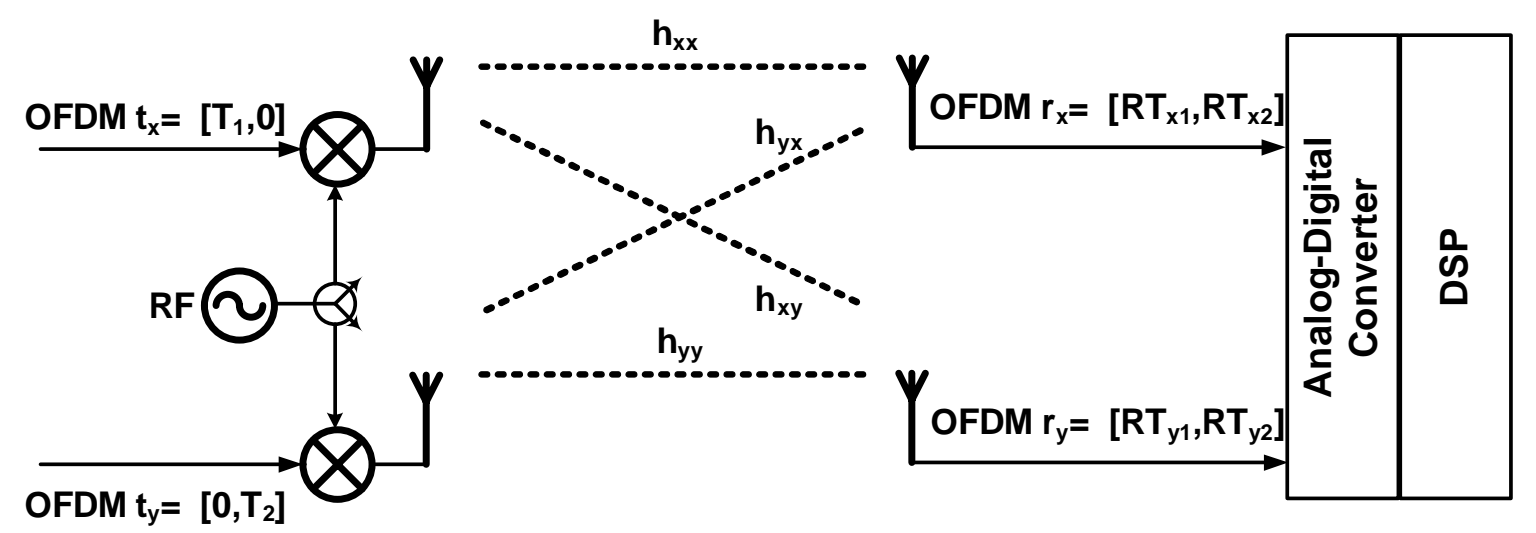

Figure 1. Block diagram of a wireless MIMO Channel

\section{TRAINING-BASED CHANNEL ESTIMATION FOR OFDM-MIMO}

In optical fiber-wireless MIMO systems, accurate channel estimation is important for signal demodulation. This is because it is the prerequisite for designing the channel equalizer in the digital domain at the receiver. In particular, when a large number of sub-carriers and advanced multiplexing technique are employed, more accurate channel estimation is required. Figure 1 shows the block diagram of a wireless MIMO OFDM channel.

In our proposed PolMux-MIMO system, the total fiber-wireless channel response for a MIMO-OFDM signal can be described as:

$$
\left[\begin{array}{l}
r_{x} \\
r_{y}
\end{array}\right]=\left[\begin{array}{ll}
h_{x x} & h_{y x} \\
h_{x y} & h_{y y}
\end{array}\right] \times\left[\begin{array}{l}
t_{x} \\
t_{y}
\end{array}\right]
$$

where $t_{x}$ and $t_{x}$ respectively stands for the transmitted signal in $\mathrm{X}$ and $\mathrm{Y}$ branch, and $t_{y}$ and $r_{y}$ are the received $\mathrm{X}$ and $\mathrm{Y}$ branch signal at the receiver. The $2 \times 2$ matrix in the equation describes the response of the fiber-wireless MIMO channel, including both the polarization mixing in the fiber and the crosstalk during wireless transmission. To obtain the parameters in the channel transfer matrix is the purpose of the channel estimation.

In order to derive this channel transfer matrix parameters, we transmit a pair of time-interleaved training sequences $T_{X}=\left[T_{1}, 0\right]^{T}, T_{Y}=\left[0, T_{2}\right]^{T}$ in the OFDM signal of the two tributaries, respectively. By precisely controlling the synchronization between the two branches' signals during the fiber-wireless transmission, assuming there is no difference in the arrival time of the two transmitted signals to the receivers in the two branches, the received training sequences in two consecutive training durations can be described as

$$
\left[\begin{array}{ll}
R T_{x 1} & R T_{x 2} \\
R T_{y 1} & R T_{y 2}
\end{array}\right]=\left[\begin{array}{ll}
h_{x x} & h_{y x} \\
h_{x y} & h_{y y}
\end{array}\right] \times\left[\begin{array}{cc}
T_{1} & 0 \\
0 & T_{2}
\end{array}\right]
$$




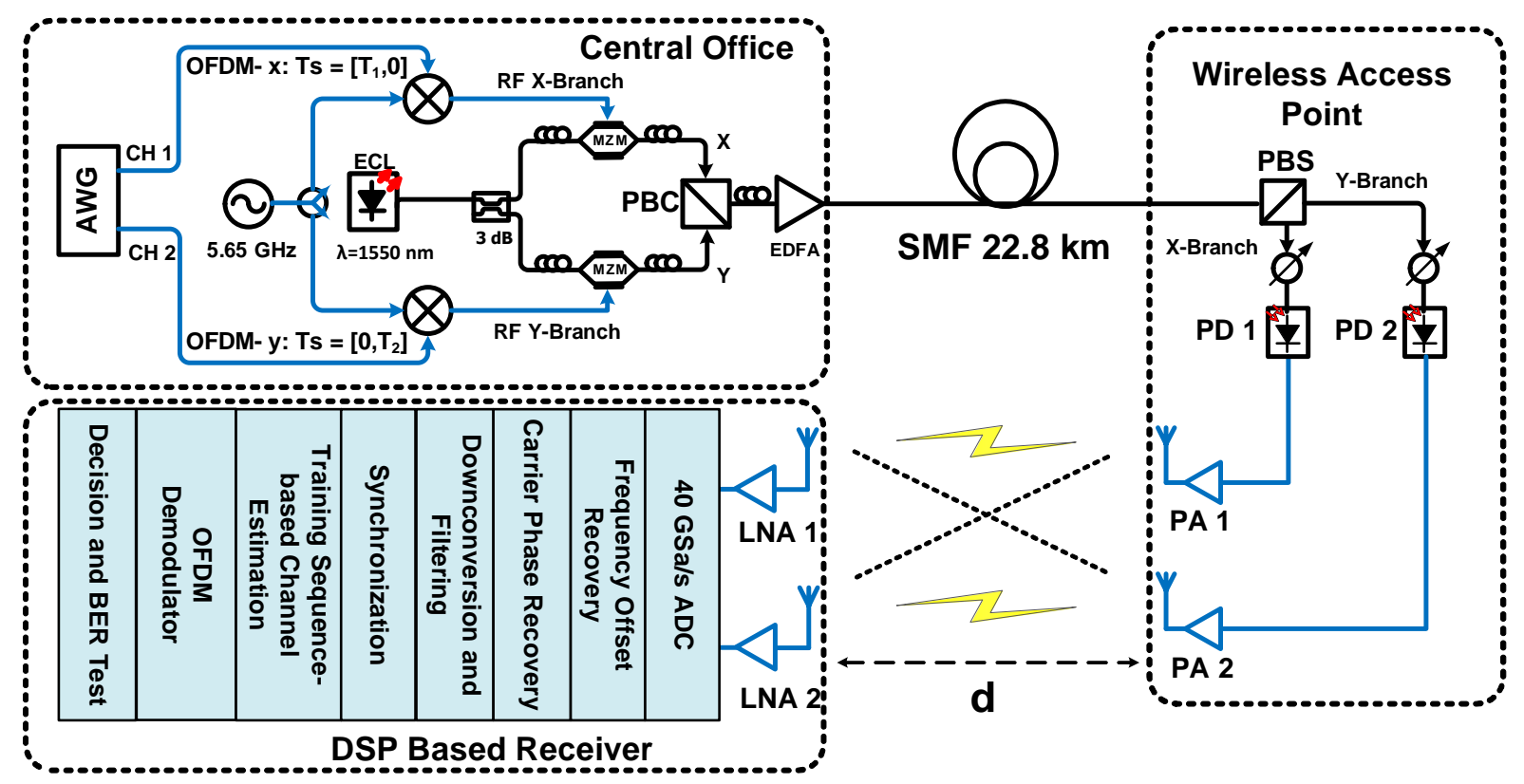

Figure 2. Experimental set-up (AWG: Arbitrary waveform generator; ECL: External cavity laser; MZM: Mach-Zehnder modulator; PBC/PBS: Polarization beam combiner/splitter; PA: Power amplifier; LNA: Low-noise amplifier)

in which $R T_{x 1}$ and $R T_{x 2}$ stands for the received training symbol from $\mathrm{X}$ branch at the first and second training durations respectively. So are the $R T_{y 1}$ and $R T_{y 2}$ for the $\mathrm{Y}$ branch. As these received training symbols can be directly obtained at the receiver side after transmission, the channel transfer matrix then can be easily calculated as

$$
\left[\begin{array}{ll}
h_{x x} & h_{y x} \\
h_{x y} & h_{y y}
\end{array}\right]=\left[\begin{array}{ll}
R T_{x 1} / T_{1} & R T_{x 2} / T_{2} \\
R T_{y 1} / T_{1} & R T_{y 2} / T_{2}
\end{array}\right]
$$

This training-based channel estimation method is computationally efficient because of its simple expression. ${ }^{8}$ On the other hand, when implementing this channel estimation method into practice, especially in the wireless MIMO systems, strict synchronization between the two channels is required. By using this method, the MIMOOFDM signals demodulation algorithm can be implemented digitally.

\section{EXPERIMENTAL SETUP}

The experimental setup for the PolMux RoF plus wireless $2 \times 2$ MIMO system is shown in figure 2 . At the RoF signal transmitter, a $1.25 \mathrm{GSa} / \mathrm{s}$ arbitrary waveform generator (AWG) is used to generate two baseband real-valued 4-QAM OFDM signals with 64 sub-carriers by an up-sampling factor of 4 . The OFDM signals have bandwidth of $625 \mathrm{MHz}$, corresponding to a gross data rate of $625 \mathrm{Mbit} / \mathrm{s}$ in each branch. The signals are arranged in frames of 10 symbols, out of which 3 are training symbols used for synchronization and channel estimation purpose. An cyclic prefix with 0.1 symbol length is added in each symbol. Therefore the signal in each $\mathrm{X}$ branch and $\mathrm{Y}$ branch has a net data rate of $397.7 \mathrm{Mbit} / \mathrm{s}$. These two OFDM signals with time-interleaved training sequences are then separately up-converted to $5.65 \mathrm{GHz}$.

A narrow line width external cavity laser (ECL) with central wavelength at $1550 \mathrm{~nm}$ is used as the lightwave source. The output optical signal is equally divided into two optical tributaries at a $3 \mathrm{~dB}$ coupler. By using 
two Mach-Zehnder modulators (MZM) which are biased at their linear points, the two RoF OFDM signals are modulated onto the optical carriers at each of the two branches. After that, by using two polarization controllers to align the polarization states of the signals at the two branches to the $\mathrm{X}$ and $\mathrm{Y}$ inputs of a polarization beam combiner (PBC), which are designed orthogonal with each other, the two signals are polarization multiplexed at the output of the PBC. A polarization controller placing after the PBC is used for roughly controlling the polarization state of the signal. The output signal then has a net data rate of $795.5 \mathrm{Mbit} / \mathrm{s}$ and thus a net spectral efficiency of $2.55 \mathrm{bit} / \mathrm{s} / \mathrm{Hz}$ is achieved. In fact, the spectral efficiency of this system is expected to be further increased if an AWG with a higher sample rate is employed and higher level modulation formats are to be used.

After being amplified by a booster erbium-doped fiber amplifier (EDFA), the PolMux RoF OFDM signal transmits through a $22.8 \mathrm{~km}$ standard signal mode fiber (SSMF). At the wireless access point, the optical signal is divided back to X and Y polarizations by a polarization beam splitter (PBS). After electrical amplification, the optical signals of these two tributaries are converted into the RF domain by two photodiodes (PD), separately, and then fed to two transmitter antennas. At the user side, two receiver antennas, placed with the same separation as the transmitter antennas, form a $2 \times 2$ wireless MIMO system. The received signals are then delivered to a $40 \mathrm{GS} / \mathrm{s}$ analog-digital convertor (ADC), where the analog signals are converted into the digital domain. A digital signal processing (DSP) based receiver, consisting of frequency and phase recovery, frequency down-conversion, training based MIMO-OFDM channel estimation and OFDM demodulation modules, is used to demodulate the received signals and evaluate bit error rate (BER).

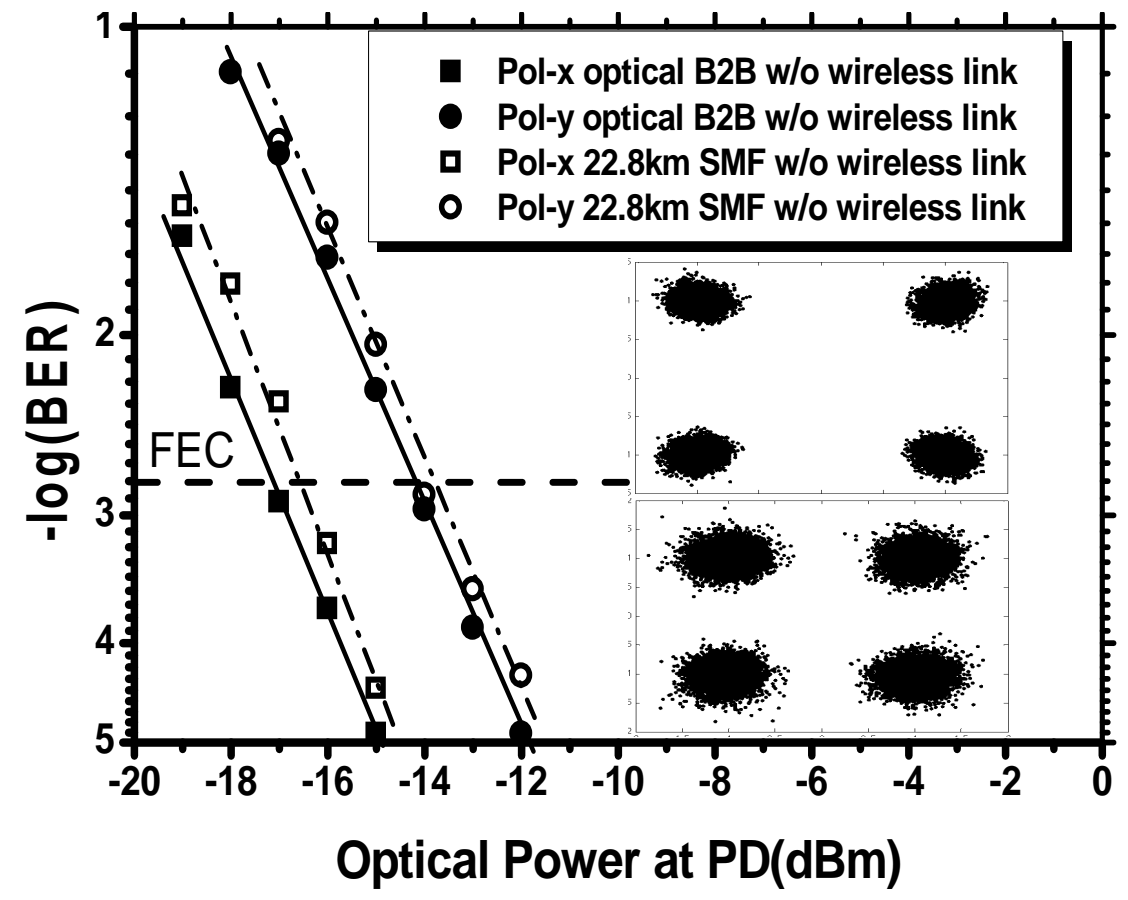

Figure 3. BER as a function of optical power at PD without wireless links (upper constellation: X polarization branch after $22.8 \mathrm{~km}$ SMF; lower constellation: Y polarization branch)

\section{RESULTS}

Figure 3 shows the tested bit-error-rate (BER) curves for the two channels' RoF signals in both optical backto-back and after $22.8 \mathrm{~km}$ single mode fiber transmission without any wireless through the air. Considering the 
FEC limit at a BER of $2 \times 10^{-3}$, from the figure it can be seen that there is around $3 \mathrm{~dB}$ difference between $\mathrm{X}$ and $\mathrm{Y}$ polarization signals. This difference is attributed to the fact that the performances of the components used during the experiment, particularly the responsivity of the two photodiodes used in the two branches are different. Apart from the difference in the performance of components, we can see that the penalty between back-to-back and fiber transmission in both branches are less than $1 \mathrm{~dB}$ at the FEC limit. The reason for such small penalty is that at low bit rate the dispersion effect in the fiber is almost negligible. Moreover, it can also be seen from the inserts of figure 3 that the received constellations of optical signals after fiber transmission in $\mathrm{X}$ branch (upper) and Y branch (below) are both clear.

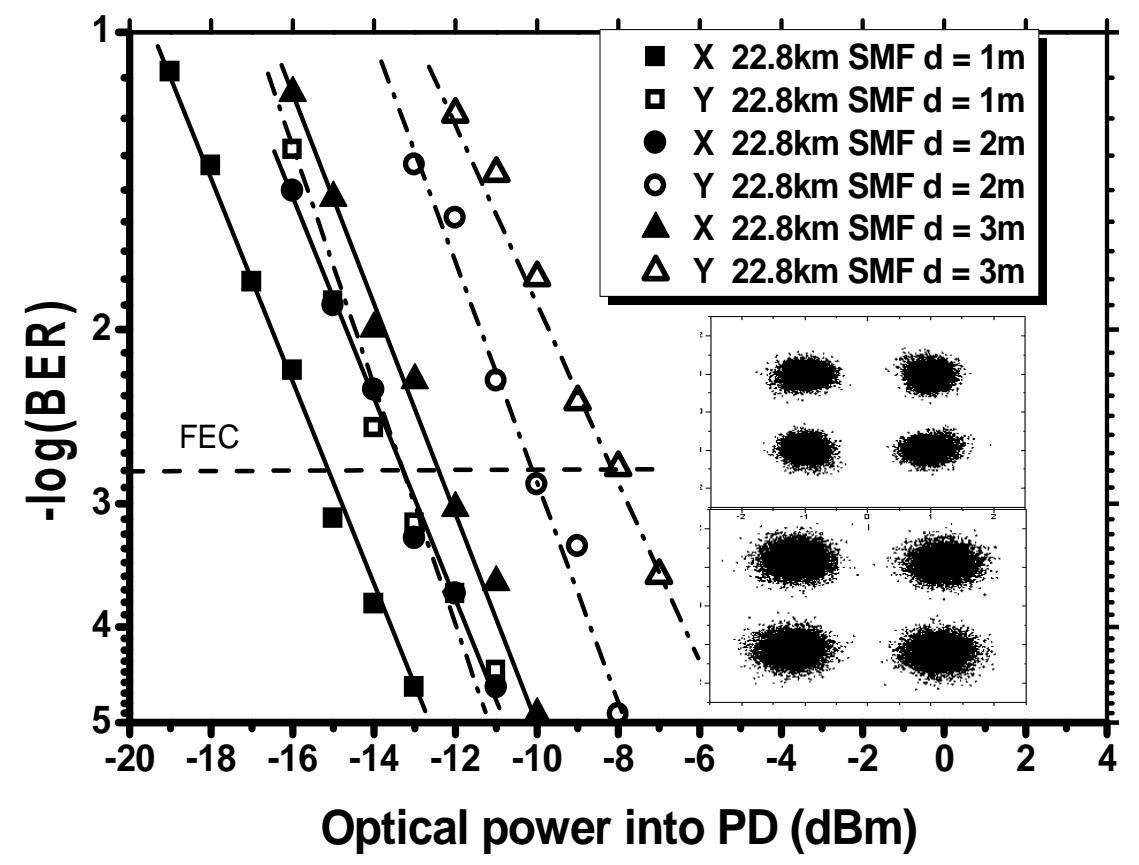

Figure 4. BER as a function of optical power at $\mathrm{PD}$ for different wireless distances (upper constellation: $\mathrm{X}$ branch at 1 $\mathrm{m}$; lower constellation: $\mathrm{Y}$ branch at $1 \mathrm{~m}$ )

The BER curves as a function of the received optical power at the photodiode after $22.8 \mathrm{~km}$ fiber for different wireless transmission distances are shown in figure 4. From the figure we can see that both $\mathrm{X}$ and $\mathrm{Y}$ channels achieve up to $3 \mathrm{~m}$ wireless transmission with BER well below the FEC limit. Moreover, the power differences between the BER curves achieving FEC limit at 1 meter wireless and 2 meters wireless are smaller than the differences between 2 meters and 3 meters wireless, for both $\mathrm{X}$ and $\mathrm{Y}$ branches. We attribute this phenomena to the more accurate channel estimation when the crosstalk angle from reference angle becomes smaller, which exceeds the influence of the decreased received RF power. The constellations of received signals after $1 \mathrm{~m}$ wireless transmission for $\mathrm{X}$ and $\mathrm{Y}$ branches are also shown in the insert of figure 4 . It can be seen that the clusters in the constellations are still clearly separated.

\section{CONCLUSION}

We propose an hybrid fiber-wireless system which seamlessly translate the optical PolMux OFDM signal into a wireless MIMO channel. The combination of the PolMux and MIMO technologies enables data transmission with a net spectral efficiency of $2.55 \mathrm{bit} / \mathrm{s} / \mathrm{Hz}$. Furthermore, a training-based scheme is digitally developed to estimate the polarization multiplexed MIMO transmission channel. A net data rate of 795.5 Mbps OFDM 
signal transmission over $22.8 \mathrm{~km}$ SMF plus $3 \mathrm{~m}$ wireless distance is successfully demodulated at the receiver for demonstration purpose. This experimental result is believed to be the first step in our ultrahigh capacity fiberwireless system research. Therefore, higher bit rate with larger bandwidth at higher frequency band together with more advanced modulation formats can be expected in our future works.

\section{REFERENCES}

[1] J. Wells, "Faster than fiber: the future of multi-Gb/s wireless," IEEE Microw. Mag, vol. 10, no. 3, pp. 104-112, 2009.

[2] Chow, C.W.; Yeh, C.H.; Wang, C.H.; Wu, C.L.; Chi, S.; Chinlon Lin; , "Studies of OFDM signal for broadband optical access networks," Selected Areas in Communications, IEEE Journal on , vol.28, no.6, pp.800-807, Aug. 2010

[3] Kumar, G.J.R.; Shaji, K.S.; , "Low complexity algorithm for channel estimation of UWB MIMO-OFDM wireless fading channels," Emerging Trends in Robotics and Communication Technologies (INTERACT), 2010 International Conference on , vol., no., pp.125-128, 3-5 Dec. 2010

[4] Alfiad, M.S.; Kuschnerov, M.; Jansen, S.L.; Wuth, T.; van den Borne, D.; de Waardt, H.; , "Transmission of 11 $\times$ 224-Gb/s POLMUX-RZ-16QAM over $1500 \mathrm{~km}$ of LongLine and pure-silica SMF," Optical Communication (ECOC), 2010 36th European Conference and Exhibition on , vol., no., pp.1-3, 19-23 Sept. 2010

[5] S.L. Jansen; I. Morita; T.C. Schenk; H. Tanaka, "Long-haul transmission of $16 \times 52.5$ Gbits/s polarizationdivision multiplexed OFDM enabled by MIMO processing", J. Opt. Netw., vol. 7, pp. 173-182, 2008.

[6] Cvijetic, N.; Prasad, N.; Dayou Qian; Ting Wang; , "Block-Diagonal MIMO Equalization for PolarizationMultiplexed OFDM Transmission With Direct Detection," Photonics Technology Letters, IEEE , vol.23, no.12, pp.792-794, June15, 2011

[7] Shu-Hao Fan; Hung-Chang Chien; Chowdhury, A.; Cheng Liu; Wei Jian; Yu-Ting Hsueh; Gee-Kung Chang; "A novel radio-over-fiber system using the xy-MIMO wireless technique for enhanced radio spectral efficiency," Optical Communication (ECOC), 2010 36th European Conference and Exhibition on , vol., no., pp.1-3, 19-23 Sept. 2010

[8] Xiang Liu; F. Buchali, "A novel channel estimation method for PDM-OFDM enabling improved tolerance to WDM nonlinearity" paper OWW5, OFC/NFOEC'2009. 